\title{
New coumarin-[60]fullerene dyads connected by an alkynyl linkage: Synthesis and fluorescence studies. Evidence for efficient singlet-singlet energy transfer
}

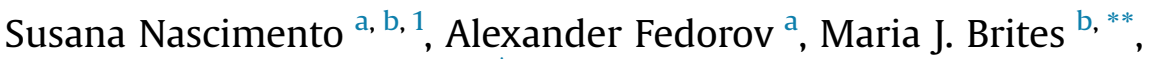 \\ Mário N. Berberan-Santos ${ }^{a, *}$ \\ a Centro de Química-Física Molecular, Instituto Superior Técnico, Universidade de Lisboa, 1049-001 Lisboa, Portugal \\ b Laboratório Nacional de Energia e Geologia, Unidade de Energia Solar, Estrada do Paço do Lumiar 22, 1649-038 Lisboa, Portugal
}

\section{A R T I C L E I N F O}

\section{Article history:}

Received 18 September 2014

Received in revised form

3 November 2014

Accepted 5 November 2014

Available online 18 November 2014

\section{Keywords:}

$\mathrm{C}_{60}$

Coumarin

Dyads

Rigid spacer

FRET

Electron transfer

\begin{abstract}
A B S T R A C T
Two new coumarin-[60]fullerene dyads, in which an alkyne group covalently links $\mathrm{C}_{60}$ to coumarin, are synthesized via 1,3-dipolar cycloaddition and spectroscopically characterized. Their photophysical properties in apolar (toluene) and polar (THF and benzonitrile) solvents are studied at room temperature, revealing the nature and polarity dependence of the excited-state interactions between the coumarin and $\mathrm{C}_{60}$ moieties. In both dyads and in all solvents, a strong quenching of the coumarin emission by $\mathrm{C}_{60}$ was observed. It mainly results from a fast and efficient singlet-singlet resonance energy transfer from the coumarin moiety to the $\mathrm{C}_{60}$ moiety, but an electron transfer contribution, enhanced in polar solvents, also exists. In toluene, the fluorescence emission of the fullerene moiety is increased by the nonradiative energy transfer process, which occurs mainly by a dipole-dipole (FRET) mechanism. In polar solvents (THF and benzonitrile), fast electron transfer from ground-state coumarin to excited fullerene is significant, leading to a partial or complete quenching of the fullerene emission, depending on the dyad.
\end{abstract}

(c) 2014 Elsevier Ltd. All rights reserved.

\section{Introduction}

The specific chemical and photophysical properties of fullerenes make them valuable building blocks for the preparation of materials with diverse applications, including solar energy conversion [1-3]. $C_{60}$, well-known as an excellent electron acceptor [4-6], has been incorporated in a large variety of molecular structures to form donor-acceptor (D-A) systems [6-8]. The excited-state donor-acceptor interactions in such systems, including photoinduced electron and energy transfer, have also been explored [9-19]. Factors such as the intrinsic properties of the individual chromophores, the interchromophoric distance, the nature of the connecting spacer, and the relative orientation are known to influence the electronic coupling between the photoactive moieties. A promising strategy to improve the electronic coupling involves

\footnotetext{
* Corresponding author.

** Corresponding author.

E-mail addresses: mjoao.brites@lneg.pt (M.J. Brites), berberan@tecnico.ulisboa.pt (M.N. Berberan-Santos).

1 Present address: Hovione Portugal.
}

donor-acceptor connection by linear $\pi$-conjugated systems [20]. Examples include oligothiophene [16,21], oligoene [11,12] and oligoyne [22-28] spacers, amongst others. In contrast to oligoenelinked D-A systems where rotation about single bonds can lead to variations in the $\mathrm{D}-\mathrm{A}$ distance and reduced conjugation within the system, in oligoynes-linked systems a fixed distance between chromophores is maintained. Thus, conformationally rigid D-A systems are obtained affording geometrically well-defined materials. The synthesis and photophysical properties of a series of flexible coumarin-fullerene $\left(C_{60}\right.$ and $\left.C_{70}\right)$ dyads were previously studied by us [15]. To further explore excited-state donor-acceptor interactions in highly conjugated and conformationally rigid $\mathrm{D}-\mathrm{A}$ systems involving coumarin and $C_{60}$, two new coumarin-[60] fullerene dyads are reported here. In these dyads, two different coumarins were covalently linked to $\mathrm{C}_{60}$ through 3-position using an alkynyl group.

\section{Experimental}

All reagents were purchased from Aldrich and AcrosOrganics (p.a. quality) and were used without further purification. The 